KUNS-1582

hep-ph/9906549

\title{
Brane fluctuations and suppression of Kaluza-Klein mode couplings
}

\author{
Masako Bando, 1, 周 Taichiro Kugo, Tatsuya Noguchi, f and Koichi Yoshioka \\ ${ }^{1}$ Physics Division, Aichi University, Aichi 470-0296, Japan \\ Department of Physics, Kyoto University, Kyoto 606-8502, Japan
}

\begin{abstract}
In higher dimensional models where the gauge and gravity fields live in the bulk and the matter fields only in a brane, we point out the importance of the brane (transverse) coordinate modes, which are the Nambu-Goldstone bosons appearing as a result of spontaneous breaking of the translation symmetry. The brane recoil effect suppresses the couplings of higher Kaluza-Klein modes to the matter, and gives a natural resolution to the divergence problem caused by the exchange of infinitely many Kaluza-Klein modes.
\end{abstract}

*E-mail: bando@aichi-u.ac.jp

${ }^{\dagger}$ E-mail: kugo@gauge.scphys.kyoto-u.ac.jp

‡E-mail: noguchi@gauge.scphys.kyoto-u.ac.jp

$\S$ E-mail: yoshioka@gauge.scphys.kyoto-u.ac.jp 
Recently, the existence of large extra dimensions beyond the usual four dimensions has been intensively investigated. This possibility can considerably lower the fundamental scale (the Planck scale [1] and/or gauge unification scale [2]) to the scales which are accessible in near future experiments due to the large volume factors and the effects of Kaluza-Klein (KK) excited states. It is also interesting that this theoretical assumption can be applied to models which may explain various phenomenological problems, such as fermion mass hierarchy [2, 3], neutrino physics [4], supersymmetry breaking [5], cosmology and astrophysics [6], and so on. In these scenarios, our world lies on a brane like a D-brane, an orientifold plane, etc., embedded in larger dimensions [2, 7].

For charged particles living on the brane which couple to a gauge field in the bulk, there has been a puzzling paradox: the amplitude for the two charged-particle scattering in the brane, caused by the gauge boson exchange, diverges if the dimensions transverse to the brane are greater than one. Indeed, the gauge interaction is naively supposed to take the form

$$
\int d^{4} x d^{\delta} y g \bar{\psi}(x) \gamma^{\mu} \psi(x) \delta^{\delta}(y) A_{\mu}(x, y)=\int d^{4} x g \bar{\psi}(x) \gamma^{\mu} \psi(x) A_{\mu}(x, 0)
$$

where $x$ and $y=\left(y^{1}, \cdots, y^{\delta}\right)$ denote the coordinates of the directions parallel and transverse to the brane, respectively, and the brane is assumed to lie at $y=0$. If we assume, for simplicity, that the transverse directions are all compactified into tori with a common radius $R$, then the gauge field is expanded into the KK modes labeled by $n$ :

$$
A_{\mu}(x, y)=\sum_{n} A_{\mu}^{(n)}(x) e^{i n \cdot y / R}
$$

Then, all the KK modes $A_{\mu}^{(n)}$ couple to the charged particle $\psi$ with equal strength $g$, and the amplitude for the two particle scattering is given by

$$
g^{2} \sum_{n} \frac{1}{-p^{2}+n^{2} / R^{2}} .
$$

But this sum over $n$ diverges when the number of the transverse dimensions $\delta$ is larger than or equal to 2 .

This is very strange. It is merely a tree-level amplitude and the divergence cannot be renormalized by any means. In the recent analyses on the experimental implications of these KK modes [8, 9, 10], the sum has simply been cut off at string scale $M_{s}$; $|n / R| \leq M_{s}$. The string scale here means the energy scale at which the detailed nature of the brane becomes relevant and the effective field theory description on the brane 
breaks down. This procedure, however, still seems strange. It is true that there exists such a built-in cutoff $M_{s}$, or the ultimate energy scale, in the present effective theory. But it is not generally true that the above expression (3) for the scattering amplitude remains valid until such an ultimate energy scale.

We here note that any type of brane necessarily fluctuates whether it originates from string theory or not. This is because there cannot exist a rigid body in the relativistic theory. Therefore there should always exist scalar fields $\phi(x)$ which stand for the coordinates of the brane in the transverse dimensions, i.e., the point $x$ in the brane occupies the point $(x, y=\phi(x))$ in the bulk. These scalar fields are Nambu-Goldstone $(\mathrm{NG})$ bosons which appear as a result of spontaneous breaking of the translational symmetry by the presence of the brane.

In this letter, we argue that if the effect of brane fluctuations, namely, the effect of these NG bosons, is correctly included, the contributions of the higher KK modes are automatically suppressed by an exponential factor and then the above problematic divergence will not actually arise.

First, we discuss the effective low-energy interactions of this NG boson field $\phi(x)$. (Hereafter, for simplicity, we consider the five-dimensional case, i.e., $\delta=1$, but it is straightforward to include more numbers of extra dimensions.) The couplings of $\phi(x)$ to the fields living only on the brane are given through the induced metric (or induced vierbein) on the brane as is well-known in string theory and described, e.g., in Ref. [11. Moreover, when some fields living in the bulk couple to the fields on the brane, $\phi(x)$ also appears in the argument $(x, \phi(x))$ of the bulk fields. For instance, the above naive interaction term (11) should be replaced by

$$
\int d^{4} x g \bar{\psi}(x) \gamma^{\mu} \psi(x) A_{\mu}(x, \phi(x))=\int d^{4} x \sum_{n} g \bar{\psi}(x) \gamma^{\mu} \psi(x) A_{\mu}^{(n)}(x) e^{i \frac{n}{R} \phi(x)},
$$

where the KK mode expansion (2) is substituted on the right-hand side. Precisely speaking, this is an approximate expression for the interaction, and the exact expression including the induced vierbein has more complicated dependence on $\phi(x)$. By examining the exact expression, however, we can confirm that the interaction (44) is indeed the leading term in neglecting all the gravitational and derivative couplings of $\phi(x)$ [12]. The kinetic term of $\phi(x)$ comes from the determinant of the induced metric, i.e., the Nambu-Goto action $\int d^{4} x\left(-\tau_{4}\right) \sqrt{-g}$, which takes the form

$$
\int d^{4} x\left(-\tau_{4}+\frac{\tau_{4}}{2} \partial_{\mu} \phi(x) \partial^{\mu} \phi(x)+\cdots\right)
$$


on the flat background. Here, $\tau_{4} \equiv f^{4} / 4 \pi^{2}$ is the brane tension and the inverse $f^{-1}$ gives a characteristic length as to how much the brane can fluctuate.

The form of the gauge interaction (4) might still seem to imply the equal couplings for all the $\mathrm{KK}$ modes $A_{\mu}^{(n)}$. However, it is deceptive. In a perturbation theory framework, it is appropriate to rewrite the exponential factor $\exp \left(i \frac{n}{R} \phi(x)\right)$ into the normal ordered form referring to the free kinetic term of $\phi$ in Eq. (5). Then the interaction term (4) takes the form

$$
\int d^{4} x g \bar{\psi}(x) \gamma^{\mu} \psi(x) A_{\mu}^{(n)}(x) e^{-\frac{1}{2}\left(\frac{n}{R}\right)^{2} \Delta\left(l_{s}\right)}: e^{i \frac{n}{R} \phi(x)}: .
$$

Here $\Delta$ is the free propagator of $\phi$

$$
\Delta(x-y) \equiv\langle\phi(x) \phi(y)\rangle=\frac{1}{f^{4}} \cdot \frac{1}{-(x-y)^{2}} .
$$

Since the present effective theory is valid only at scales larger than $l_{s}=M_{s}^{-1}$, the propagator $\Delta(x)$ with $|x| \leq l_{s}$ is understood to be $\Delta\left(l_{s}\right)=1 / f^{4} l_{s}^{2}$, and we have replaced the infinite $\Delta(0)$ by the value $\Delta\left(l_{s}\right)$ in Eq. (6). This form of the interaction term (6) now implies that the effective coupling $g_{n}$ of the level $n$ KK mode to four-dimensional fields is actually suppressed exponentially:

$$
g_{n} \equiv g \cdot e^{-\frac{1}{2}\left(\frac{n}{R}\right)^{2} \frac{M_{s}^{2}}{f^{4}}} .
$$

Note that the origin of this suppression is a recoil effect of the brane. This is easily seen if we note that the effective couplings $g_{n}$ can also be written as

$$
g_{n}=g \cdot\left\langle 0\left|e^{i \frac{n}{R} \phi(x)}\right| 0\right\rangle
$$

in the cutoff theory. In this expression $|0\rangle$ is the (free theory) ground state of $\phi$ field corresponding to a Gaussian wave functional peaked at $\phi(x)=0$. Remembering that $\phi(x)$ is the transverse coordinate, the operator $e^{i \frac{n}{R} \phi(x)}$ is just like the vertex operator in the string theory and gives transverse momentum $n / R$ to the brane around the point $x$. Hence, $e^{i \frac{n}{R} \phi(x)}|0\rangle$ represents the recoiled state of $\phi$ by the absorption (emission) of the KK mode $A_{\mu}^{(n)}$ carrying transverse momentum $n / R(-n / R)$. Thus the amplitude $\left\langle 0\left|e^{i \frac{n}{R} \phi(x)}\right| 0\right\rangle$ can be viewed as a probability of containing the original state $|0\rangle$ in the recoiled state $e^{i \frac{n}{R} \phi(x)}|0\rangle$. As is clear from this view, the suppression becomes stronger for higher KK modes since larger deformation of the brane occurs, and on the other hand, in case of the stiff brane possessing large $f$, the suppression is weak. 
Now, with this new form of interaction term (6), let us reconsider the problem of the charged-particle scattering amplitude discussed above. With the coupling (6), the contribution of the level $n$ KK modes is estimated as

$$
\begin{aligned}
\mathcal{A}_{n} & =g_{n}^{2} \cdot\left\langle A_{\mu}^{(n)}(x) A_{\nu}^{(-n)}(0)\right\rangle\left\langle: e^{i \frac{n}{R} \phi(x)}:: e^{-i \frac{n}{R} \phi(0)}:\right\rangle \\
& =g^{2} e^{\left(\frac{n}{R}\right)^{2}\left(\Delta(x)-\Delta\left(l_{s}\right)\right)} \cdot\left\langle A_{\mu}^{(n)}(x) A_{\nu}^{(-n)}(0)\right\rangle .
\end{aligned}
$$

Note that we here have a factor of effective coupling function $g^{2} e^{\left(\frac{n}{R}\right)^{2}\left(\Delta(x)-\Delta\left(l_{s}\right)\right)}$. It has an interesting property that it interpolates from the suppressed value to the "bare" value:

$$
g^{2} e^{\left(\frac{n}{R}\right)^{2}\left(\Delta(x)-\Delta\left(l_{s}\right)\right)}= \begin{cases}g_{n}^{2} & \text { for }|x| \gg l_{s}, \\ g^{2} & \text { for }|x| \simeq l_{s} .\end{cases}
$$

When we measure the effective 4-fermion coupling at energy scale much smaller than the cutoff scale $M_{s}$, therefore, the KK modes contribute to it as if they have the suppressed gauge couplings $g_{n}=g \cdot \exp \left[-\frac{1}{2}(n / R)^{2}\left(M_{s}^{2} / f^{4}\right)\right]$. With this exponential factor damping rapidly with $n$, the higher KK mode contributions become smaller and the infinite sum of those modes surely converges as it should be. N Note that this convergence remains to hold even when the transverse dimensions are greater than one, since the exponential damping factor appears for each extra dimension.

When the brane is hard enough, $f \sim M_{s}$, the cutoff for the KK mode sum in the present treatment reduces to the usual naive cutoff at $|n / R| \sim M_{s}$ because $g_{n} / g$ remains to be $\sim 1$ until the highest KK modes with mass $\sim M_{s}$. However, since we now wish to consider the effects of the brane fluctuation, we assume $f<M_{s}$. For example, if we take $f \sim R^{-1}\left(\ll M_{s}\right)$, then $g_{n} / g \sim e^{-n^{2}\left(M_{s} R\right)^{2}}$ which is almost zero for all non-zero KK modes. In this way, owing to this large exponential suppression factor with moderate value of brane tension $f$, all the KK contributions from the tree-level graphs can be suppressed.

We discuss some phenomenological implications of this suppression factor. Suppose that the standard model gauge fields live in the bulk while the quarks and leptons live on the brane. Then, as we have calculated above, the effective 4-Fermi coupling constant $G_{F}$ receives corrections from the KK excitation modes. The correction is dominated by the first mode contributions $\mathcal{A}_{ \pm 1}$ in Eq. (10) since the higher modes are further

${ }^{*}$ A similar exponential factor is also discussed in a string framework with orbifold compactification [13. 
suppressed by the exponential factors, so that the KK mode correction $\Delta G_{F}$ to the 4-Fermi coupling constant can be estimated roughly as

$$
\frac{\Delta G_{F}}{G_{F}} \sim \frac{\mathcal{A}_{+1}+\mathcal{A}_{-1}}{\mathcal{A}_{0}}=2 \frac{g_{n=1}^{2}}{g^{2}} \frac{M_{W}^{2}}{M_{W}^{2}+1 / R^{2}} .
$$

Since the standard model prediction precisely agrees with experiments, the KK mode correction (12) must be small, say $\lesssim O\left(10^{-2}\right)$ [14. We consider $R^{-1}$ in the range $O(100 \mathrm{GeV})<R^{-1}<M_{s}$, then the constraint $\Delta G_{F} / G_{F}<10^{-2}$ reads

$$
2 M_{W}^{2} R^{2} \cdot e^{-\frac{M_{s}^{2}}{R^{2} f^{4}}}<10^{-2} .
$$

This gives a weak constraint to the brane tension $f$. We show in Fig. 1 the allowed region of $f$ for several values of $M_{s}$. Clearly, since $\exp \left[-\left(M_{s}^{2} / R^{2} f^{4}\right)\right]<1$, no constraint appears if $R^{-1}$ is larger than $10 \sqrt{2} M_{W} \sim 1.1 \mathrm{TeV}$. Even for $R^{-1}$ less than this, Fig. 1 shows that the constraint (13) can be satisfied for any $M_{s}$ provided that $f$ is chosen suitably small. Therefore, if the brane fluctuation is taken into account, the constraints on the extra dimensions $\left(R, M_{s}\right)$ so far obtained can be substantially loosened. It is clear from the above discussion that when there are $\delta(\geq 2)$ extra dimensions, the suppression factor becomes $\left(g_{n} / g\right)^{2 \delta}$ and the constraint of $f$ is further weaker.

This suppression of the KK mode couplings to four-dimensional fields also works in the loop diagrams as well as in the tree diagrams. This effect may be serious and forces reconsideration of the scenarios proposed so far. Consider, for example, the model proposed in Ref. [2] in which the minimal supersymmetric standard model gauge fields and Higgs doublets live in the bulk and the other matter fields are confined on our four-dimensional brane. In this model, the three gauge couplings evolve up with power-law behavior and rapidly unify at rather low-energy scale. In addition, Yukawa couplings were also expected to have the power-law running behaviors, which could give an interesting origin of the fermion mass hierarchies. However, the power-law running behaviors were due to the contributions of KK modes of gauge and Higgs fields in the loop diagrams. If we take the effect of the brane fluctuation into account, the Yukawa couplings no longer show the power-law running, unfortunately, since the relevant loop diagrams always contain the couplings between bulk fields and four-dimensional ones which are suppressed exponentially for the higher KK modes. On the other hand, since the gauge fields live in the bulk and their self-interactions are totally irrelevant to the brane fluctuation, the running of the gauge couplings still obeys the power law, and the $\mathrm{TeV}$-scale grand unification scenarios can remain valid. 
Finally, we comment on the suppression of the contributions from KK gravitons. The effects of the KK gravitons have been expected to be observable in the low-scale quantum gravity scenarios $\left(M_{s} \sim O(\mathrm{TeV})\right)$ and many authors have investigated the relevant phenomena observable in the near future collider experiments and in astrophysics and cosmology [8, 9]. Similarly to the case of the bulk gauge fields, the contributions of the higher KK gravitons are also suppressed. But this time the compactification radius $R$ should be very large: $O(\mathrm{~mm})-O(\mathrm{fm})$, depending on the number of the compactified extra dimensions, $\delta$. Because of this large radius, the mass spectrum of the KK modes is nearly continuous and the sum over the KK momenta can be replaced by an integration. For example, consider the process of matter scattering on the brane by the exchange of an infinite number of KK gravitons. The amplitude $\mathcal{M}$ takes the following form, omitting the numerator factors unimportant to the present discussion:

$$
\begin{aligned}
\sum_{n} \frac{1}{M_{P}^{2}} \frac{e^{-\left(\frac{n}{R}\right)^{2} \frac{M_{s}^{2}}{f^{4}}}}{\left(\frac{n}{R}\right)^{2}-t} & =\frac{R^{\delta}}{M_{P}^{2}} \int d^{\delta} k e^{-k^{2} \frac{M_{s}^{2}}{f^{4}}} \int_{0}^{\infty} d \tau e^{-\left(k^{2}-t\right) \tau} \\
& =\frac{\pi^{\frac{\delta}{2}}}{M_{s}^{\delta+2}} \int_{0}^{\infty} d \tau e^{t \tau}\left(\tau+\frac{M_{s}^{2}}{f^{4}}\right)^{-\frac{\delta}{2}},
\end{aligned}
$$

where $t(<0)$ is the momentum transfer $\left(|t| \gg R^{-2}\right), 1 / M_{P}^{2}$ is the graviton coupling constant, and the relation $M_{P}^{2}=M_{s}^{\delta+2} R^{\delta}$ has been used. Note that the presence of the exponential suppression factor $\exp \left[-(n / R)^{2}\left(M_{s}^{2} / f^{4}\right)\right]$ has made it possible to integrate over the KK momentum without artificial cutoff. When $\sqrt{|t|} \ll f^{2} / M_{s}$, the amplitude (14) approximately becomes

$$
\begin{aligned}
\mathcal{M} & \sim \frac{1}{M_{s}^{4}} \ln \left(\frac{f^{4}}{-t M_{s}^{2}}\right) & (\delta=2), \\
& \sim \frac{1}{M_{s}^{\delta+2}}\left(\frac{f^{2}}{M_{s}}\right)^{\delta-2} & (\delta>2) .
\end{aligned}
$$

In the case of $f \simeq M_{s}$, this expression reduces to the usual result by the simple cutoff taking no account of the brane fluctuation. If the tension $f$ is smaller than $M_{s}$, however, we see that the cross section $\sigma$ for this process is suppressed as

$$
\sigma \simeq\left(\frac{f}{M_{s}}\right)^{4(\delta-2)} \times \sigma\left(f=M_{s}\right)
$$

for $\delta>2$. Because of the large power $4(\delta-2)$, the anticipated observable effects from KK gravitons are, unfortunately, strongly suppressed with moderately small values of 
$f / M_{s}$, and then no new constraint on the fundamental scale $M_{s}$ may be imposed from these arguments. The case of two extra dimensions, $\delta=2$, is exceptional, where the suppression effect by the brane fluctuation is only logarithmic and the effects of KK gravitons may still be observable.

In conclusion, we have discussed the effects of brane reactions to the emission and absorption of the KK modes. With a generic value of the brane tension $f<M_{s}$, the couplings of higher KK modes to the matter on the brane are exponentially suppressed by the brane recoil effect and then the problematic divergence in summing up those contributions is automatically avoided. When the brane tension $f$ becomes smaller, the KK mode couplings becomes weaker and hence the detection of their signatures becomes more difficult. This might seem unwelcome from a phenomenological viewpoint. However, since $1 / f$ plays the role of the coupling constant of the brane coordinate NG mode $\phi$, it will become easier to detect $\phi$ produced copiously in various processes 12. We hope the future experiment will check the existence of $\phi$, which is a direct evidence for our world to lie on a brane.

\section{Acknowledgments}

The authors would like to thank K. Hashimoto, T. Hirayama, H. Kawai and M. Yamaguchi for many valuable discussions and comments. M. B. and T. K. are supported in part by the Grants-in-Aid for Scientific Research No. 09640375 and No. 10640261 , respectively, from the Ministry of Education, Science, Sports and Culture, Japan, and K. Y. is supported in part by the Grant-in-Aid for JSPS Research Fellowship.

\section{References}

[1] I. Antoniadis, Phys. Lett. 246B (1990) 377; J.D. Lykken, Phys. Rev. D54 (1996) 3693; N. Arkani-Hamed, S. Dimopoulos and G. Dvali, Phys. Lett. 429B (1998) 263; I. Antoniadis, N. Arkani-Hamed, S. Dimopoulos and G. Dvali, Phys. Lett. 436B (1998) 257; N. Arkani-Hamed, S. Dimopoulos and J. March-Russell, hepth/9809124.

[2] K.R. Dienes, E. Dudas and T. Gherghetta, Phys. Lett. 436B (1998) 55; Nucl. Phys. B537 (1999) 47. 
[3] S. Abel and S. King, Phys. Rev. D59 (1999) 095010; N. Arkani-Hamed and S. Dimopoulos, hep-ph/9811353; N. Arkani-Hamed and M. Schmaltz, hep-ph/9903417; H.-C. Cheng, hep-ph/9904252; K. Yoshioka, hep-ph/9904433.

[4] K.R. Dienes, E. Dudas and T. Gherghetta, hep-ph/9811428; N. Arkani-Hamed, S. Dimopoulos, G. Dvali and J. March-Russell, hep-ph/9811448; A.E. Faraggi and M. Pospelov, Phys. Lett. 458B (1999) 237; G. Dvali and A.Yu. Smirnov, hep-ph/9904211.

[5] I. Antoniadis, C. Muñoz and M. Quirós, Nucl. Phys. B397 (1993) 515; E.A. Mirabelli and M.E. Peskin, Phys. Rev. D58 (1998) 065002; I. Antoniadis, S. Dimopoulos, A. Pomarol and M. Quiros, Nucl. Phys. B544 (1999) 503.

[6] N. Arkani-Hamed, S. Dimopoulos and G. Dvali, Phys. Rev. D59 (1999) 086004; M. Maggiore and A. Riotto, Nucl. Phys. B548 (1999) 427; N. Kaloper and A. Linde, Phys. Rev. D59 (1999) 101303; N. Arkani-Hamed, S. Dimopoulos, N. Kaloper and J. March-Russell, hep-ph/9903224; hep-ph/9903239; A. Riotto, hep-ph/9904485.

[7] G. Shiu and S.H.H. Tye, Phys. Rev. D58 (1998) 106007; Z. Kakushadze and S.H.H. Tye, Nucl. Phys. B548 (1999); Z. Kakushadze, Nucl. Phys. B552 (1999) 3.

[8] G.F. Giudice, R. Rattazzi and J.D. Wells, Nucl. Phys. B544 (1999) 3; S. Nussinov and R. Shrock, Phys. Rev. D59 (1999) 105002; E.A. Mirabelli, M. Perelstein and M.E. Peskin, Phys. Rev. Lett. 82 (1999) 2236; T. Han, J.D. Lykken and R.-J. Zhang, Phys. Rev. D59 (1999) 105006; J.L. Hewett, Phys. Rev. Lett. 82 (1999) 4765; P. Mathews, et al., Phys. Lett. 450B (1999) 343; ibid. 455B (1999) 115; hep-ph/9904232; hep-ph/9905395; T.G. Rizzo, Phys. Rev. D59 (1999) 115010; M.L. Graesser, hep-ph/9902310; K. Cheung and W.-Y. Keung, hep-ph/9903294; K. Cheung, hep-ph/9904266.

[9] N. Arkani-Hamed, S. Dimopoulos and G. Dvali, in Ref. [6]; S. Cullen and M. Perelstein, Phys. Rev. Lett. 83 (1999) 268; L.J. Hall and D. Smith, hep-ph/9904267; V. Barger, T. Han, C. Kao and R.-J. Zhang, hep-ph/9905474; X.-G. He and G.C. Joshi and B. McKellar, hep-ph/9908469.

[10] P. Nath and M. Yamaguchi, hep-ph/9902323, hep-ph/9903298; M. Masip and A. Pomarol, hep-ph/9902467; 
[11] R. Sundrum, Phys. Rev. D59 (1999) 085009.

[12] M. Bando, T. Kugo, T. Noguchi and K. Yoshioka, in preparation.

[13] I. Antoniadis and K. Benakli, Phys. Lett. 326B (1994) 69.

[14] Particle Data Group, Eur. Phys. J. C3 (1998) 1. 


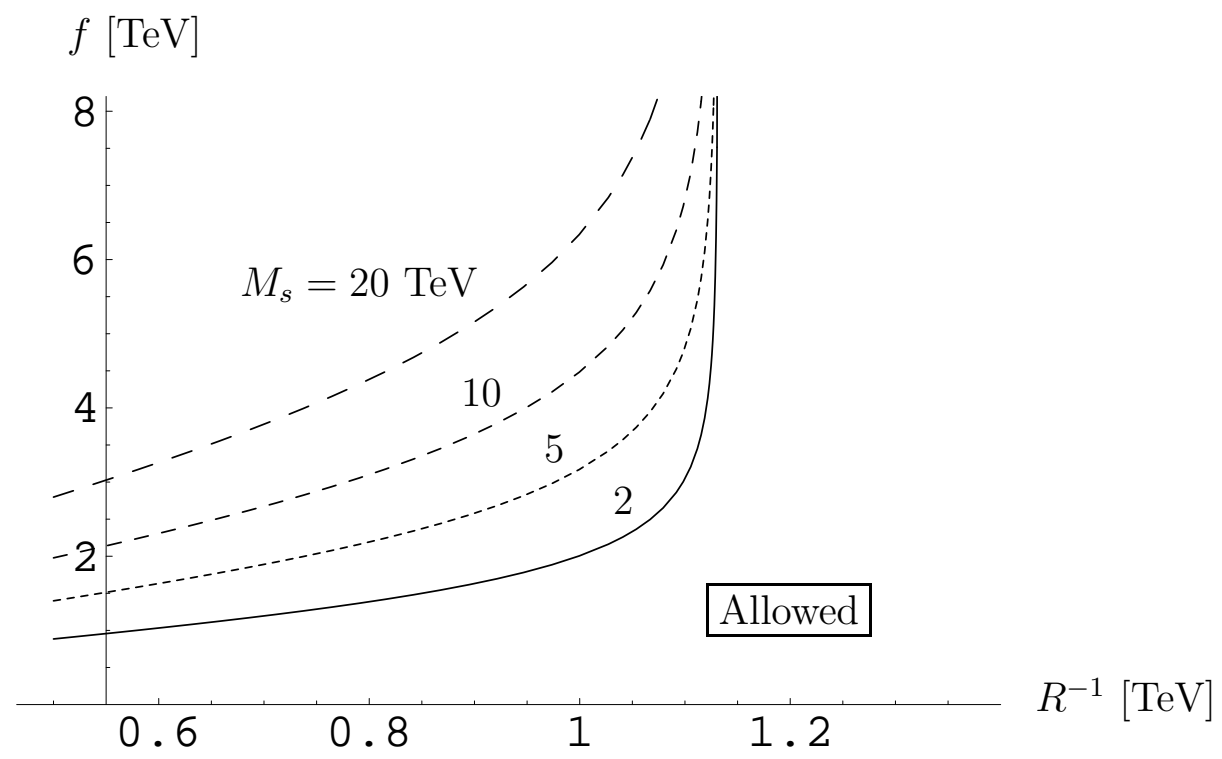

Figure 1: Typical allowed region for brane tension $f$ from the measurement of the Fermi constant. The region below each line is allowed for each value of $M_{s}$. For $R^{-1} \gtrsim 1.1$ $\mathrm{TeV}$, there exists no constraint on $f$. 